\title{
1 Reporting Suspected Abuse or Neglect in Research Involving Children
}

3

4

\section{Abstract}

In this article, we explore the ethical issues related to the reporting of suspected abuse or neglect in research involving children. Ethical dilemmas related to reporting child maltreatment are often complex because the rights of children and their adult caregivers may conflict and determinations of abuse or neglect are socially constructed judgments that depend on particular circumstances. We argue that when reporting is legally mandated, investigators must follow the law and report their suspicions to Child Protective Services. When reporting is not legally mandated, investigators still have an ethical obligation to report to help prevent additional maltreatment and allow children to obtain access to services needed to recover from abuse or neglect. We also argue that investigators should include plans and procedures in the research protocol for making reports and training research staff in recognizing evidence of child abuse or neglect. Although investigators should report evidence of abuse or neglect that is discovered incidentally, they have no mandate to actively search for such evidence when it is not related to the study's objectives. Investigators should also inform parents and children about their obligations to report suspected abuse or neglect.

\section{Introduction}

Maltreatment is a serious medical and psychosocial problem that impacts millions of children each year. In 2014, 3.2 million children in the U.S. were the subjects of at least one report of abuse or neglect to a Child Protective Services (CPS) agency and 1,580 children died from abuse or neglect. The most common forms of reported child maltreatment included neglect (75\%), physical abuse (17\%) and sexual abuse (8.3\%). ${ }^{\mathrm{i}}$ Professionals made $63 \%$ of the reports, while 37\% were made by non-professionals or were not classified.[1] In addition to producing acute pain and suffering, maltreatment can cause long-term psychological and physiological harm, including impaired brain development, learning and cognition; anxiety, depression; borderline personality disorder; drug and alcohol abuse; chronic illnesses; and increased risks of juvenile delinquency and criminality.[2,3]. 
Most countries have laws against child maltreatment and have adopted international

2 declarations affirming children's rights, including the right not to be neglected or abused.[4],[5]

3 Although our paper focuses primarily on the U.S. legal context as we wanted to focus on ethical

4 debates, rather than international comparisons, the conclusions we draw and the suggestions we

5 make have implications for all researchers, as human ethical arguments transcend national

6 boundaries. Moreover, US and non-U.S. researchers are likely to face the same ethical dilemmas

7 which arise from the intergenerational power dynamic, between children and adults, in all

8 societies. .

9 Some of the international agreements include: the United Nations Declaration of the Rights of

10 the Child (1959), the United Nations Convention on the Rights of the Child (1989), the African

11 Charter on the Rights and Welfare of the Child (1990), and the European Convention on the

12 Exercise of Children’s Rights (1996).[6]

13 In the U.S., 56 states or territories require certain professionals (such as social workers, 14 teachers, physicians, or nurses) to report suspected child abuse or neglect, while 18 require certain professionals and any person who suspects child abuse or neglect to report it.[3]). Most states distinguish between four types of mistreatment of children: physical abuse, sexual abuse, psychological abuse, and neglect.[1] Some states require that reporting be based on a reasonable suspicion but do not specify the degree or type of evidence needed to make this judgment.[1] Evidence could include suspicious bruises, cuts, burns, scars, or broken bones or verbal reports from children.

Reporting suspected child maltreatment can pose ethical challenges for physicians (and other health care professionals) because they may be uncertain whether abuse or neglect has occurred and are wary of making a false report, which could cause the family significant, distress and disruption and damage their relationship with the physician.[7] A key issue in deciding whether to make a report is whether they have sufficient evidence to justify making a report and physicians must rely on their professional judgment and experience when making this

27 determination. [7]

Although much has been written about ethical dilemmas related to research involving children and reporting child maltreatment, very little has been written about the conjunction of these topics.[8] Investigators who are conducting research involving children can face reporting 
1 that oversee research involving human subjects, such as Institutional Review Boards (IRBs) or

2 Research Ethics Boards (REBs), can encounter dilemmas related to their responsibilities to ensure that research is conducted ethically. In this article, we will explore the ethical issues that

4 investigators and oversight committees face related to reporting of suspected abuse or neglect

5 involving children as research subjects when child maltreatment is not the primary focus of the study, i.e. suspected abuse or neglect is discovered incidentally.

\section{The Ethical Basis for Reporting Suspected Abuse or Neglect}

Investigators have legal duties to report suspected child maltreatment in many jurisdictions, but in others they do not. In a state that only requires certain types of professionals to report child abuse or neglect, an investigator may have no legal duty to make a report if he or she is not a member of one of the mandated professions (e.g. the investigator is an exercise physiologist but the law only applies to social workers, educators, physicians, and nurses). We argue, however, that investigators still have ethical duties to report, even when the law does not specifically require them to do so.

The primary ethical rationale for reporting suspected abuse or neglect is to safeguard the welfare of the child by preventing him or her from experiencing additional harm and providing him or her with access to ongoing support for recovery from maltreatment.[10] When an investigator makes a report to the local CPS, the agency can investigate the case and take effective action to protect the child, such as placing him or her in temporary protective custody. CPS can also help the child obtain treatment for acute medical or psychiatric problems. Once a report occurs, the investigator's responsibilities end as CPS and the legal system take over. A court may then hear the case and determine whether the child should be returned to the family or placed in more permanent protective custody, e.g. foster care. Courts also have the power to restrict or terminate parental rights and mandate mental health treatment for parents who abuse or neglect their children. The courts make their decisions by considering how to promote the best interests of the child, while keeping in mind the importance of family reunification, if appropriate.[11]

Human research regulations and ethical guidelines do not explicitly deal with reporting child maltreatment, but they require investigators to safeguard the welfare of human research 
1 subjects, which implies a duty to report suspected child abuse or neglect, if ones assumes that reporting child maltreatment is likely to protect child research participants from harm.[12-16] Including a plan to address suspected child maltreatment in a study protocol for research involving children would help to ensure that participants are protected from harm, but, as we

5 shall see below, needless harms could occur to the family if reporting is not handled properly.[17] So, the comply with regulations and guidelines research should strive to protect the child and his or her family. subject or his or her parent or guardian, reporting child maltreatment therefore involves breaching the confidentiality of research participants and their parents, which raises its own ethical and legal issues (discussed below). Research regulations and ethical guidelines require human studies to include provisions for protecting the confidentiality and privacy of participants.[12-16] If the research is conducted in a clinical setting, such as a hospital or reporting when child protection laws do not require them to report. Several factors contribute to this complexity. First, legal requirements focus on the rights of research participants or patients, not on those of adult caregivers (i.e. parents or guardians), and do not address the issue how to settle conflicts between parental rights to privacy and confidentiality and children's rights to protection from harm. Second, determinations of abuse or neglect are socially constructed judgments that often depend on the child's and parents' particular circumstances [8] Third, researchers may not understand exceptions to confidentiality laws that allow them to report suspected abuse or neglect, nor how to manage the conflicts that arise between the rights of children and their adult caregivers. Fourth, researchers may be uncertain as to whether confidentiality laws prohibit them from reporting if they are unfamiliar with these legal requirements. Most confidentiality in the U.S. and other countries include exceptions that permit

27 breaches in specific circumstances, such as reporting certain types of infectious diseases to public health departments or reporting suspected abuse or neglect.[18] To deal with these complex issues, researchers should understand the arguments for and against reporting.[19] 
2 Arguments Against Reporting

Although reporting suspected maltreatment would likely benefit the research subject, some researchers argue that reporting often has the opposite effect and can cause harm to parents

5 and other family members.[200] Kotch discuses child maltreatment reporting issues related to research that he and his colleagues conducted on stress and social support in mother-child pairs at risk for child maltreatment. The study, which followed the pairs from the child's birth,

8 included interviews with the mothers and children at ages 6, 8, and 12.[9] The investigators

9 blinded the interviewers to the children's answers to sensitive questions most likely to raise suspicions of abuse or neglect. The investigators took this extraordinary step to protect the confidentiality of the participants and avoid triggering a legal obligation to report suspected abuse or neglect. Kotch cites four reasons for trying to avoid legal reporting mandates: 1) reports violate confidentiality; 2) reports often cause harm to the child due to retaliation from an angry parent; 3) most reports are unsubstantiated, which causes the family unnecessary stress and disruption; and 4) CPS is often ineffective at protecting children from harm.[9] Despite the investigators' efforts to blind interviewers, five out of 442 children (0.1\%) participating in follow up interviews shared information that led to CPS reports. Only one of these reports was substantiated.[9]

Let's examine each of these arguments. Concerning the first argument, as noted earlier, most confidentiality laws allow reporting of abuse or neglect. While reports to CPS do violate ethical duties of confidentiality, one might argue that it is acceptable to breach confidentiality to prevent serious, imminent harm to identifiable individuals.[21] A famous U.S. court case, Tarasoff v. Regents of the University of California, addressed this issue.[22] In this case, 24 Prosenjit Poddar, a University of California at Berkeley student, told his psychotherapist, Dr. Lawrence Moore, that he wanted to kill another student, Tatiana Tarasoff, when she returned from a trip. The psychotherapist informed the campus police about this threat and recommended

27 that Poddar be civilly committed because he was suffering from a mental illness (schizophrenia) and was dangerous to himself or others. The police briefly detained Poddar but released him after determining that he was not dangerous. Neither Moore nor the campus police warned

30 Tarasoff about Poddar's threat. Tragically, Poddar stabbed Tarasoff to death when she returned from her trip. Tarasoff's parents sued the university and Dr. Moore for negligence, arguing that 
failing to warn Tarasoff about the threat violated their obligation to exhibit reasonable care to

2 protect her from harm. The defendants appealed the case to the California Supreme Court after lower courts ruled in favor of the plaintiffs (i.e. Tarasoff's parents). The California Supreme

4 Court ruled in favor of the plaintiffs, arguing that the professional duty of confidentiality does

5 not allow one to ignore threats to public health and safety.[22]

Although the Tarasoff case addressed legal, not ethical duties, and involved a verbal threat not mere suspicion or harm, one might argue that the rationale for breaching

8 confidentiality is the same in both contexts. The strength of the obligation to breach confidentiality should depend on the professional's judgment concerning the probability and magnitude of the potential harm that can be prevented. If the professional has convincing evidence of potential harm that is serious and imminent, breaching confidentiality is warranted. If the evidence is weak and the harm is neither serious nor imminent, then breaching confidentiality may not be warranted.[21]

With regard to breaching confidentiality, a researcher's main task is to decide whether he or she suspects abuse or neglect has occurred.[5] If a researcher judges that he or she has sufficient evidence to warrant this judgment, his or her primary responsibility is to report suspected maltreatment to CPS, which may review the evidence, interview the child and parent(s), and decide what actions should be taken to protect the child's welfare.

Education and training on applicable definitions of abuse and neglect and how to recognize these problems in the context of family dynamics plays a key role in enabling researchers and staff members to make these difficult, socially constructed judgments.[8]

22 Without adequate training a student or inexperienced researcher may not have the required knowledge to make a competent choice concerning the decision to report suspected abuse or neglect, which could lead to reporting when there is no evidence of abuse or neglect or failing to report when there is. Training should include lectures on the legal, ethical, and practical issues related to reporting suspected maltreatment; standard operating procedures for reporting; and

27 discussions of case scenarios.[8]

Turning to the second argument, we agree that researchers should be concerned about potential retaliation against the child. However, retaliation would involve the actions of the parent(s) and responsibility for such violence and or emotional abuse should be attributed to the perpetuator(s). If CPS is unable to protect children, this would be a social and political issue that 
researchers may wish to pursue, but the responsibility to safeguard children would remain with the child's parent(s) and the state, but not with the researchers.

Regarding the third argument, we agree that researchers should try to avoid causing innocent parents and other family members unnecessary stress and disruption, which is one reason why it is so important to provide investigators and staff members with education and training in recognizing and responding abuse or neglect.[5] Training and education will enable researchers to recognize the signs of abuse or neglect and make appropriate referrals Concerning the fourth argument, while we agree that researchers should be concerned that CPS interventions are often ineffective, it is not the researcher's responsibility to ensure that CPS does its job properly. Researchers can discharge their legal and ethical duties by reporting their suspicions to CPS and trusting that the agency will handle the situation properly.

\section{Informed Consent}

Human research regulations and ethical guidelines require investigators to provide participants (or their legal representatives) with information pertinent to the decision to enroll in a study during the consent process.[12-16] Regulations and guidelines also require investigators to provide children who are capable of assenting with study information tailored to their level of understanding. ${ }^{\text {ii }}$ [12-14] Although regulations and guidelines focus on disclosing information to human subjects, one might argue that investigators should inform parents about their obligations to report suspected child abuse or neglect, because these would be risks the parents would undertake when their children participate in research.[8,23] Investigators should inform children about their reporting requirements during the assent process.

Some commentators have argued that investigators may be wary of informing parents about their obligations to report suspected child abuse or neglect because this may lead some parents to not to allow their children to participate in the study, which could interfere with enrollment.[9,23] Informing parents about reporting obligations could also damage the trust between investigators and participants which is essential to the conduct of research.[23] Although parents who have maltreated their children may be most fearful of mandated reporting, even parents who have not harmed their children could be concerned about dealing with an unsubstantiated report of abuse or neglect.[9,23] However, we think it is important to note that 
empirical data do not support this argument because studies have shown that informing parents about reporting obligations does not undermine recruitment or enrollment.[8,24]

While we can understand why parents would want to avoid false accusations of abuse or neglect, investigators have little choice but to tell parents about their reporting obligations so that the parents can make informed choices.[23] One strategy for dealing with this disclosure dilemma is to inform parents (and assenting children) about obligations to report child abuse or neglect only for studies in which there is a reasonable chance that investigators will acquire evidence of child maltreatment. For example, if a study only requires children to perform simple cognitive tasks, there is very little chance that investigators will acquire evidence of abuse or neglect; if a study includes a physical exam, there would be a reasonable chance of acquiring evidence of abuse or neglect, because investigators might detect suspicious bruises, lacerations, or scars. A study that involves interviews or surveys on sensitive topics (such as mental health, sexual activity, or drug/alcohol use) would also have a reasonable chance of uncovering evidence of child maltreatment. Furman University, for example, only requires researchers to include mandated reporting language in the consent document when there is a reasonable chance that they will acquire information about child abuse or neglect.[25]

One might argue, however, that researchers should always notify parents about mandated reporting laws because researchers and IRB members may not understand whether a study has a reasonable chance of producing evidence of maltreatment. Moreover, researchers may have a bias toward claiming that their study does not have a reasonable chance of producing evidence of maltreatment because they do not want to undermine enrollment by disclosing reporting requirements. The University of California at Berkeley's IRB, for example, requires that all studies involving children include mandated reporting language in consent and assent forms.[26] Accordingly, we believe that the norm of transparency requires researchers to always inform children and parents about reporting mandates.[27,28]

If investigators inform parents (and assenting children) about mandated reporting, they should do so in a non-threatening way that assures them that their confidentiality and privacy will be protected to the full extent allowed by law. 
2 Should Researchers Actively Look for Evidence of Abuse or Neglect?

Although we think that in most situations researchers have an ethical obligation to report suspected child maltreatment when they happen to acquire evidence of it, we do not think that researchers have an obligation to look for evidence of maltreatment when that is not part of the study’s objectives, since doing so would require the researcher to describe new objectives, develop standard operating procedures for reporting suspected maltreatment, and train study

8 staff, all of which would require expenditures of additional time, effort, and money. If the researcher has a limited budget and support staff, then allocating additional funds to actively searching for evidence of maltreatment could take away funds from other important research activities, which could have a negative impact on the overall rigor and utility of the study.

For example, suppose that a psychologist is planning to conduct a study that is not likely to produce evidence of suspected abuse or neglect, such as an experiment that asks elementary school children to perform simple cognitive tasks like memorizing words. Should the psychologist actively look for evidence of abuse or neglect by, for example, including sensitive survey questions in the study? Based on the arguments outlined above, we think the answer is

\section{Research Oversight Issues} oversight committees, such as IRBs or REBs. When oversight committees review research involving children, they must decide whether the protocol should include plans and procedures for reporting suspected abuse or neglect. Plans and procedures should account for imbalanced power relationships between children in the study and their adult caregivers. The committee must also decide whether consent and assent documents should include language that describes mandated reporting of abuse or neglect and how language should be worded for adult caregivers and child participants. When research occurs at a site in which investigators (or other members of the research team) have legal obligations to report, the committee has little choice but to require investigators to follow the law. A more difficult choice arises when there are no legal obligations to report. In this situation, the committee should require investigators to include plans and procedures for reporting child maltreatment to benefit research participant (see 
1 discussion above). To ensure that reporting is handled properly and to minimize risks of

2 erroneous reports, the committee should also require investigators to include plans for training research staff in recognizing suspected abuse or neglect as well as standard operating procedures

4 for handling reports, i.e. when to report, how to report, whom to report to, and so on.[8] The 5 committee should also require investigators to inform them of reports in a timely fashion. A 6 report of abuse or neglect could be treated as an unanticipated problem arising in research, an 7 adverse event, or both. ${ }^{\text {iii }}$ The committee could use this information to decide whether to require 8 the investigator to take additional steps, such as modifying the protocol or standard operating 9 procedures, to promote the welfare of research participants. To avoid potential delay and confusion about reporting procedures, investigators should report suspected maltreatment to CPS

11 before informing the IRB or REB

\section{Conclusion}

Reporting evidence of suspected child maltreatment obtained during research involving children presents ethical dilemmas for investigators and oversight committees. When reporting is legally mandated, investigators must follow the law. When reporting is not legally mandated, investigators have an ethical obligation to report suspected abuse or neglect to help prevent additional maltreatment and allow children to access resources needed to recover from maltreatment. They should also include plans and procedures in the research protocol for making reports and training research staff in recognizing evidence of child abuse or neglect. Although investigators should report evidence of abuse or neglect that is discovered incidentally, they have no obligation to actively look for such evidence when it is not related to the study's objectives. Investigators should inform parents and children about their obligations to report suspected abuse or neglect Oversight committees that review research involving children should require investigators to describe their plans and procedures for reporting child abuse or neglect in the protocol, including their plans for training research staff and informing parents and

27 children about their reporting obligations. Additional studies on reporting evidence of abuse or neglect discovered in research involving children may help investigators and oversight committees decide how to handle reporting dilemmas and address parents' and children’s concerns. 


\section{References}

1. Administration for Children and Families. Child Maltreatment 2014. Available at: http://americanspcc.org/wp-content/uploads/2014/03/2014-Child-Maltreatment.pdf. Accessed: 8 March 2017.

2. Child Welfare Information Gateway. Long-Term Consequences of Child Abuse and Neglect. Available at:

4. Cutland M. Child abuse and its legislation: the global picture. Arch Dis Child 2012; 97(8):679-684.

5. Australian Government, Australian Law Reform Commission. Family Violence: A National Legal Response, published 11 November 2010. Available at: https://www.alrc.gov.au/publications/family-violence-national-legal-response-alrc-report114. Accessed: 18 December 2017.

6. Library of Congress. Child's Rights: International Laws. Available at: https://www.loc.gov/law/help/child-rights/international-law.php. Accessed: 27 April 2017.

7. Shanley JR, Shropshire JD, Bonner BL. To report or not to report: a physician's dilemma. Virtual Mentor 2009;11(2):141-145.

8. Randall D, Anderson A, Taylor J. Protecting children in research: Safer ways to research with children who may be experiencing violence or abuse. J Child Health Care 2015;20(3):344-353.

9. Kotch JB. Ethical issues in longitudinal child maltreatment research. $J$ Interpers Violence 2000;15(7): 696-709.

10. Urquiza A. Yes, there should be mandated reporting for researchers. Monitor on Psychol 2003;34(6):28. embers who engage in serious violence. Journal of Gang Research 8(4): 35-56. 
11. Jones WG. Working with the Courts in Child Protection. Department of Health and Human Services, Administration for Children and Families. Available at: https://www.childwelfare.gov/pubPDFs/courts.pdf. Accessed: 23 March 2017.

12. Department of Health and Human Services. Protection of Human Subjects. Title 45 Code of Federal Regulations 46 (2009).

13. Department of Homeland Security, Department of Agriculture, Department of Energy, National Aeronautics and Space Administration, Department of Commerce, Social Security Administration, Agency for International Development, Department of Justice, Department of Labor, Department of Defense, Department of Education, Department of Veterans Affairs, Environmental Protection Agency, Department of Health and Human Services, National Science Foundation, and Department of Transportation. Final Rule: Federal Policy for the Protection of Human Subjects. Federal Register 2017;82(12):7149-7274.

14. Emanuel EJ, Wendler D, Grady C. 2000. What makes clinical research ethical? JAMA 2000;283(20):2701-2711.

15. National Commission for the Protection of Human Subjects of Biomedical and Behavioral Research. The Belmont Report: Ethical Principles and Guidelines for the Protection of Human Subjects of Research. Washington, DC: U.S. Department of Health, Education, and Welfare, 1979.

16. World Medical Association. Declaration of Helsinki-Ethical Principles for Medical Research Involving Human Subjects, 2013 revision. Available at: https://www.wma.net/policies-post/wma-declaration-of-helsinki-ethical-principles-formedical-research-involving-human-subjects/. Accessed: 19 April 2017.

17. Randall D, Childers-Bushle K, Anderson A, Taylor J. An analysis of child protection 'Standard Operating Procedures for research' in Higher Education Institutions in the United Kingdom. BMC Med Ethics 2015;16(1):66.

18. Yarmohammadian MH, Raeisi RA, Tavakoli N, Nansad LG. Medical record information disclosure laws and policies among selected countries; a comparative study. J Res Med Sci 2010; 15(3):140-149.

19. Taylor J, Spencer N, Baldwin N. Social, economic and political context of parenting. Arch Disease Childhood 2000; 82(2): 113-120. 
20. Uttal D. 2003. No, researchers should not be mandated reporters of child abuse. Monitor on Psychol 2003;34(6):29.

21. Beauchamp TL, Childress JF. Principles of Biomedical Ethics, 7th ed. New York: Oxford University Press, 2012.

22. Tarasoff v. Regents of the University of California. 1976. 17 Cal. 3d 425, 551 P.2d 334, 131 Cal. Rptr. 14 (Cal. 1976).

23. Sieber JE. Issues presented by mandatory reporting requirements to researchers of child abuse and neglect. Ethics Behav 1994;4(1):1-22.

24. Stiffman A, Brown E, Striley CW, Ostmann E, Chowa G. Cultural and ethical issues concerning research on American Indian youth. Ethics Behav 2005;15(1):1-14.

25. Institutional Review Board, Furman University. Informed consent template, 15 April 2009 version. Available at: http://www.furman.edu/sites/IRB/information/Documents/Informed_Consent_Template \%20.doc. Accessed: 31 March 2017.

26. Committee for the Protection of Human Subjects, University of California at Berkeley. 2016. Mandated reporting for suspected child, elder, or dependent adult abuse or neglect, May 2016. Available at: http://cphs.berkeley.edu/mandated_reporting.pdf. Accessed: 31 March 2017.

27. Gondolf EW. 2000. Human subject issues in batterer program evaluation. J Aggress Maltreat Trauma 2000;4(1): 273-297.

28. Carroll-Lind J, Chapman JW, Gregory J, Maxwell G. The key to the gatekeepers: passive consent and other ethical issues surrounding the rights of children to speak on issues that concern them. Child Abuse Negl 2006;30(9): 979-989.

\section{Notes}

\footnotetext{
i The numbers add up to more than $100 \%$ because some children were victims of more than one type of maltreatment.

ii Assent to research participation is different from consent. Consent is a form of giving legally valid permission to some activity, whereas assent is only an agreement and acknowledgment with no legal implications. The age for giving valid consent varies across different countries.

iii Federal regulations require institutions to have procedures for reporting anticipated problems and serious or continuing non-compliance to the IRB.
} 